$\xi=$ 줄

\title{
Telescopic double crowns in prosthodontics
}

\author{
$\operatorname{Recep~Kara}^{1 *}$ \\ ${ }^{1}$ Dental Protheses Technologies Department, Istanbul Aydin University, Istanbul 34295 TURKEY \\ *Corresponding author E-mail: drecepkara@gmail.com
}

\begin{abstract}
The purpose of this article is to classify the types of telescopic prostheses that protect natural teeth as an alternative to traditional removable prostheses, and to explain their advantages, disadvantages, indications and clinical significance in order to minimize the problems of removable dentures. Telescopic prostheses consist of an inner (primary) crown permanently cemented to an abutment and an outer (secondary) crown attached to the prosthesis. Primary crowns protect the abutment from bruises and thermal irritation, and also provide retention and stabilization of the secondary crown. The secondary crown combines the primary crown with the prosthesis to form a telescopic unit, providing prosthetic retention and stability.
\end{abstract}

Keywords: Overdenture, Denture Retention, Dental Crown, Removable Partial Denture

\section{Introduction}

This Many different systems are improved for fixed partial dentures as a retention element in the prosthetic rehabilitation of partial edentulism [1,2]. At the beginning of the 20th century, telescopic crowns began to be used as retainers for removable partial dentures (RPDs). They are also known as double crown and sleeve coping or Conusrone, a German term that describes the cone-shaped design. Telescopic crowns have mainly been used in RPDs to attach prostheses to the remaining teeth. [3,4]. But they can also be described as retainers in detachable prostheses entirely of abutment origin [5]. Telescopic or conical crowns are widely used for retention. high comfort, long-term survival can be achieved with these crowns [6].

A telescope crown was discovered in 1873 by Dr. Patented by J. B. Beers [7]. The telescopic bridge business The American System of Dentistry published a removable bridge system supported by telescope crowns in 1887 and F. A. Peeso in 1894 [8,9]. Telescopic crowns are widely used in some European countries such as Germany and Sweden and eastern Asia [9].

The term telescopic prosthesis refers to the type of prosthesis that includes double crowns as a retainer or attachment. These retainers consist of two crowns; a primary or inner crown cemented to the abutment and a secondary or outer crown connected to the prosthesis (Fig. 1) [10,11]. These crowns consist of an inner or primary telescopic coping permanently cemented to an abutment and a compatible removable outer or secondary telescopic crown rigidly attached to a removable prosthesis. Copings were designed to protect the abutment from caries and thermal irritation, and also provided retention and stabilization of the secondary crown. The secondary crown attaches to the primary coping to form a telescopic unit and acts as a retainer for the rest of the tooth structure. The conical configuration of the contacting walls produces a compressive inter-surface tension due to the wedge action. This concept is widely used in engineering for fast and safe fixing of two matched mechanical parts. The tension must be strong enough to retention the RPD in place. In restorations, the retention and stabilizing properties of telescopic retainers, which include the entire dental arch, are directly related to their numbers, wall angles, and their harmonious distribution along the dental arch.

The average taper angle is usually 6 degrees $[3,4]$. Wear-off of the primary crown walls reduces the adhesion with the secondary crown. The smaller the taper degree, the greater the friction retention [3]. If the abutment height is smaller, which affects the size of the intersurface contact between both components, a reduced angle of 2 to 5 degrees on each side should be used to improve retention. The splinting effect of telescopic restorations occurs when multiple outer and fixed inner telescopic crowns are joined together in place. Splinting and support elements are not affected by the degree of wall angulation [5].

Telescopic attachment dentures are functionally comparable to traditional fixed partial dentures (FPDs) and are considered the most effective replacement for lost teeth and are well tolerated psychologically. The distribution of abutments for detachable prostheses must comply with the principles governing the production of FPDs. Accordingly, removable prostheses are generally only indicated for patients with multiple abutments distributed bilaterally in strategic positions along the dental arch.

Telescopic crowns have also been used successfully in RPDs and FPDs supported by endosseous implants in combination with natural teeth, including overdentures $[12,13]$. The primary advantage of a telescopic prosthesis is retrievability. If the remaining dentition is in a 
state of transition, abutments splinted with FPDs can be a problem. A telescopic prosthesis is a more versatile alternative for these patients because the prosthesis can be repaired without reconstruction of the entire superstructure, despite a localized failure.

The patient can distinguish the telescopic restoration by the extending of the outer telescopic crowns from their copings. The patient should be instructed to avoid damage to the prosthesis during cleaning, as the distortion of the outer telescopic crown may render the prosthesis dysfunctional. There is ample evidence that telescopic prostheses improve oral hygiene and periodontal health because abutments are more accessible for oral hygiene. Also, the peripheral gingival margins and prosthesis can be easily cleaned after removal from the mouth $[3,13]$. The splinting effect of the telescopic superstructure is similar to an FPD and has a positive effect on the stabilization of the remaining tooth structure and improves periodontal health. This clinical report describes the clinical and technical aspects of the use of telescopic crowns in the restorative treatment of two patients.

The outer surface of the seconder crown can have the anatomical shape of the natural tooth [14] or it can be in a simple coping shape without anatomical landmarks $[9,15]$. Retention is achieved by fitting the outer crown to the inner crown $[11,15,16]$.

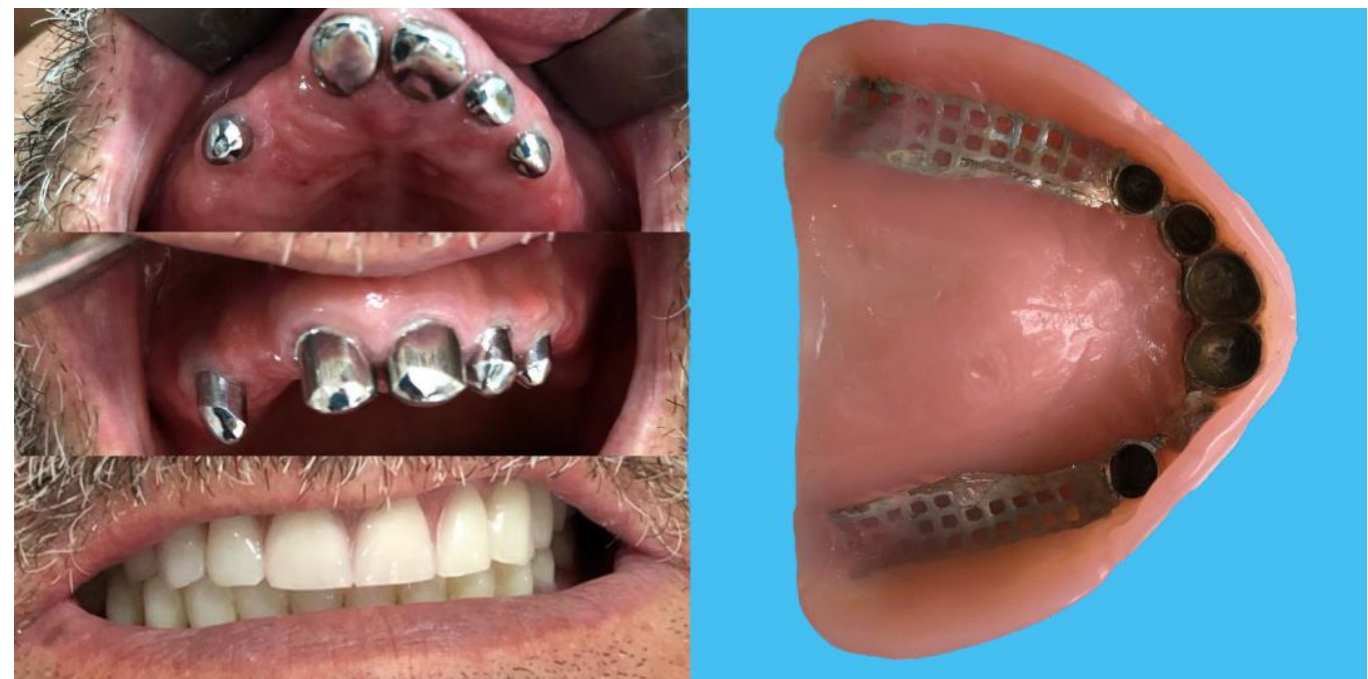

Fig. 1: Telescopic crowns (over teeth and implants)

\section{Types of telescopic crowns}

Types of telescopic crowns Many types of telescopic crowns have been produced: 1-) Rigid double-crowns (cylindrical, conical) 2-) Non-rigid double-crowns (resilient designs), 3-) Modified designs

\subsection{Rigid double-crowns}

Rigid double-crown-retained RPDs are defined as those with no clearance fit between inner and outer crowns. The RPDs use friction or a wedging effect between crowns for exerting retentive forces. These included conically prefabricated copings with wedging effect, frictional telescopic crown, and galvanoformed (electroformed) outer crowns (Fig. 2).
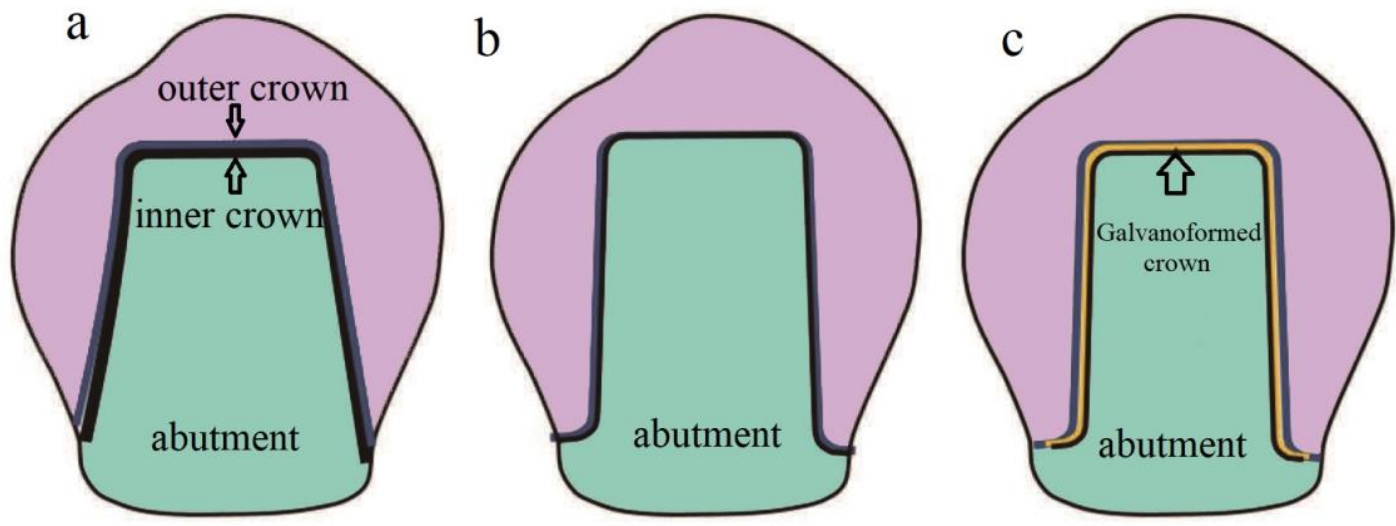

Fig. 2: Rigid telescopic crowns

\subsubsection{Cylindrical crowns}

This type is a telescopic crown form with a parallel-sided inner crown (Fig. 3A) [17]. Retention is achieved by friction between the inner and outer crowns [18]. This design was developed by Haupl and Böttger as stated by Langer and Behr [6,18]. The cylindrical design has excellent retention and good aesthetic advantages in the marginal area $[6,19]$. However, these crowns are very difficult to 
manufacture because a perfect and correct fit between crowns is required [19]. Besides, as a result of continuous friction, the wear rate of metal surfaces increases, and a leverage movement occurs [16]. Therefore, this type can only be used in abutments with soundsupporting texture when a lot of retention is needed.

\subsubsection{Conical crowns}

This strain is a modification of the previous system and was developed by K. H. Körber as noted by Langer, Hulten, Shiba, and Behr [3,6,20]. The inner crown is cone-shaped (Fig. 3B) [19]. Thus, their axial surfaces are tapered occlusally at a certain angle called the convergence angle (or taper) [16,21]. The retention is achieved with a wedge action. The smaller the angle of convergence, the greater the retention force $[3,6,10]$. An angle of convergence of $6^{\circ}$ is recommended. It results in a retention force of 5-10 N [6]. Shiba mentioned that the $4^{0}-8^{0}$ angle can be used according to the crown length and physiological movement of the abutment [22]. Some authors have proposed a $2^{0}$ angle to maintain accepted retention $[19,21,23]$.

The conical type is more commonly used than the cylindrical design as it is less difficult to manufacture and is less harmful to abutments and support tissues [16,21,23]. This design also has the advantage of determining the forces to be applied to each abutment by choosing the angle of convergence according to the clinical situation [3]. However, the disadvantage of the conical design is that retention is reduced after a period of use [19,23,24].
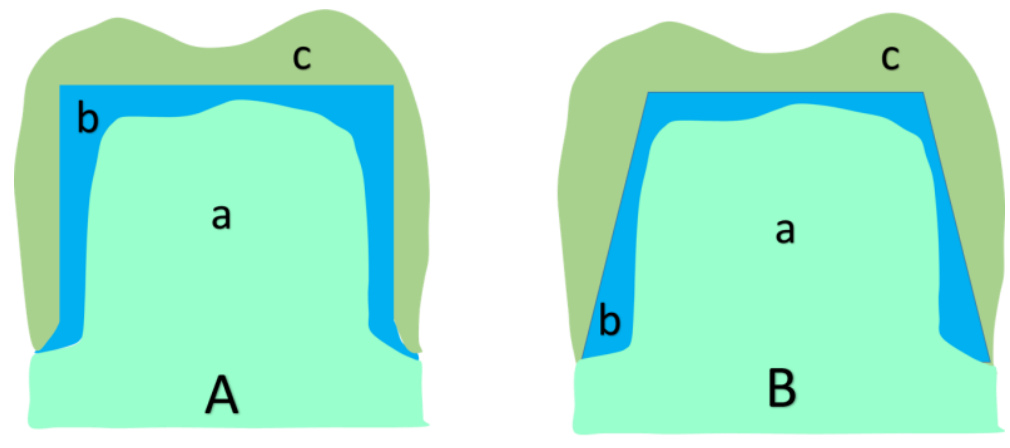

Fig. 3: A:Cylindrical crown, B:Conical crown

\subsection{Non-rigid double-crown (Resilient Designs)}

Non-rigid types have a gap between the inner and outer crowns at the terminal position of a prosthesis. There is no holding force due to the space between the crowns. An additional holding attachment is needed, including the Marburg double-crown TK-SNAP and the friction pin on the hybrid telescopic crown (Fig. 4).

Since they allow some freedom in vertical and rotational movements between inner and outer crowns, they can be called non-rigid designs [4][3]. This can be achieved with some changes to the inner crown, outer crown, or both. These modifications result in reduced close contact and creating a gap between the inner and outer crowns.

The Marburg double crown is a well-known flexible design [25,26]. It is based on a gap fit system where only the cervical third of the inner crown is parallel to the outer crown and provides a space between the crowns. This gap allows little lateral movement between the crowns and prevents stress generation $[25,27,28]$. In the Hofmann and Ludwig design, the cervical half of the inner crown is parallelsided, while the occlusal semi-conical and there is a gap of 0.2-0.5 mm between the crowns in the occlusal region [3][4]. Yalisove presented a conical design in which the contact between the crowns was limited to 2 occlusal thirds, with a 76-254 $\mu \mathrm{m}$ gap between the crowns in the cervical third, allowing the outer crown to rotate and prevent unwanted friction [28].

These designs provide a flexible relationship between the abutment and the prosthesis and, according to the introducer and its advocates, prevent harmful effects, adapt to the elasticity of the tissues, provide better force distribution and increase the survival rates of the abutments. Flexible designs can be advantageous in cases of few residual or weak fulcrums and remote extensions. Studies have shown that the resistant design is successful when used in implant-supported prostheses $[29,30]$.

\subsection{Modified Designs}

Some systems have been developed with significant changes to the double crown concept. Often they depend on combining the telescopic system with another type of connection:[2]

1-) Magnotelescopic crowns [2].

2-) O-ring coping attachment [5].

3-) Prefabricated telescopic attachments [3,31]. 


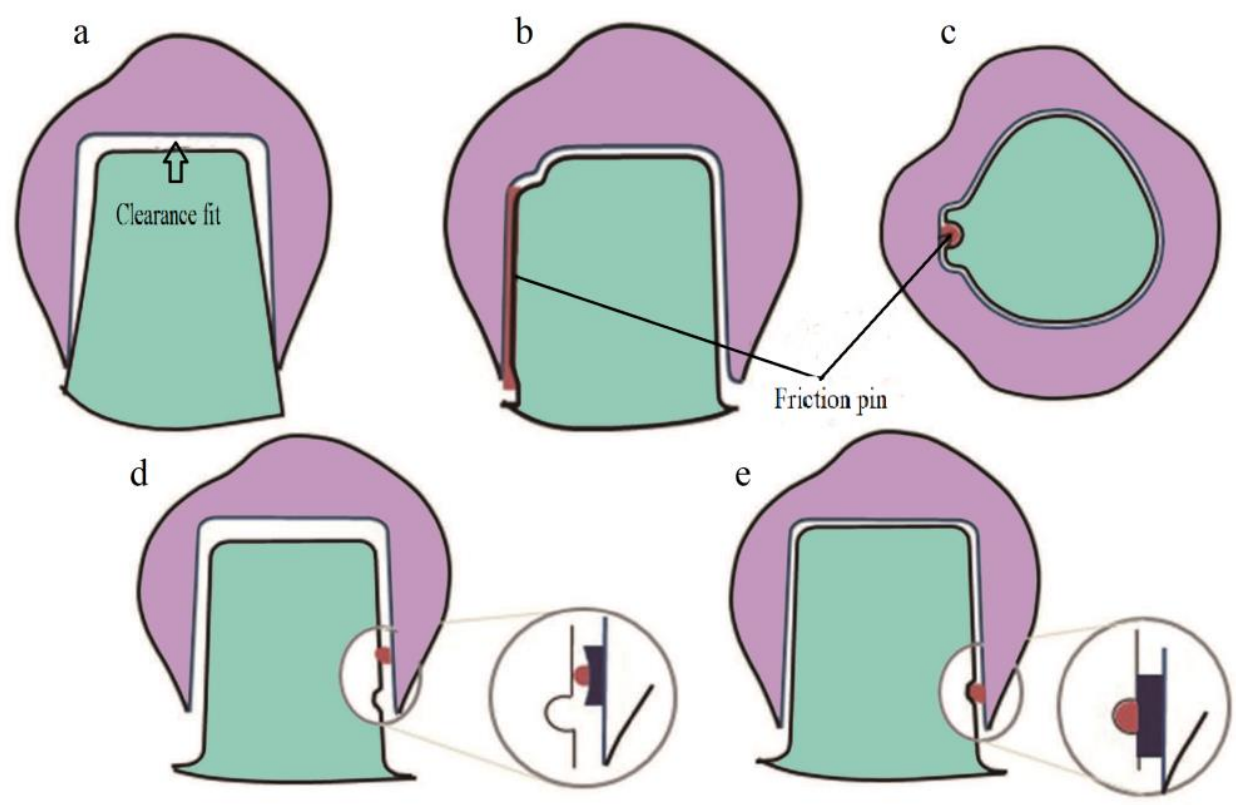

Fig. 4: Resilient telescopic crowns

\section{Telescopic Crown Advantages}

The large retention force results from the engagement and extensive contact between the surfaces of the inner and outer crowns $[4,8,9,19,32,33]$. This is due to the correct relationship between the inner and outer crowns as they are firmly attached to the prosthetic base $[8,10,32]$. Since telescopic crowns completely surround the abutments, occlusal forces are transferred to the abutments via their long axis $[4,32]$. The entryway for the prosthetic seating is easily provided by the parallelism of the inner crowns, even if the abutments are inclined [5,33]. The telescopic attachment provides effective home care and good oral hygiene by providing access to the gum tissues of the abutment. Moreover, a good fit of the inner crown to the abutment protects it from bruises and thermal irritation [3]. The use of double crowns as a retentive element provides better aesthetics than clamps [32]. Good aesthetics can be achieved with ceramic surfaces and appropriate color selection. Many authors have reported good patient satisfaction rates with telescopic prostheses [5,8-10,33]. Telescopic prostheses can be easily repaired even if an abutment is lost $[5,10,21,34]$.

\section{Telescopic Crown Disadvantages}

Telescopic prosthesis fabrication requires very complex clinical and laboratory procedures. This results in a long treatment time and increased cost $[3,33]$. It can be difficult to achieve the exact retention required between two crowns. Moreover, prosthetic retention can only be evaluated after cementation of the crowns [11]. Also, after a period of use, the retention force between the crowns decreases. This is due to repeated insertion and removal of the prosthesis and the wear of the crown metal $[19,24,35,36]$. Failure to ensure proper fit of crowns or poor oral hygiene can result in cervical caries [20,21]. Aesthetic problems and difficulties can arise, such as showing the plated metal or excessive contouring of the crowns.[33] Technical failure is one of the major problems with the telescopic prosthesis. There may be technical failures such as loss of cementation, loss of veneer, or breakage of the artificial teeth, metal frame, or prosthetic base [6]. Several studies have reported a high rate of technical failure with this type of prosthesis $[6,17,21,36]$. Follow-up, periodic evaluation, and maintenance are required to overcome problems with technical malfunctions, cervical caries, and retention [37][21].

\section{Clinical uses and indications of telescopic crowns}

Telescopic prostheses are widely used clinically. There are many different types and designs of the double crown and telescopic prostheses [4,32]. They are generally used in the rehabilitation of mouths where a few teeth remain. These dentures can be produced with acrylic base $[8,9]$.

In addition to being used with natural teeth, telescopic prostheses can be augmented with implants [29,30]. Moreover, telescopic prostheses may be a good option for attaching implants to natural teeth [38]. The indications for telescopic crowns are generally as follows [2].

a-) Situations where there is a small number of abutment teeth without proper distribution $[5,21,39]$.

b-) Abutment teeth should be covered with crowns due to extensive caries or poor contour [4,32].

c-) Abutment teeth with protected prognosis $[8,21,40]$.

d-) Advanced cases of periodontitis [20,39,41].

e-) When it is difficult to find a suitable way of insertion, such as with unique abutment teeth [16][5][33].

f-) Oral cancer patients [39,42].

g-) Attachment of natural teeth to implants [43].

h-) Occlusal reconstruction cases [2].

i-) Patients with poor dexterity [2,27]. 


\section{Discussion}

Telescope crowns were introduced in the early 20th century as a attachment for movable partial dentures (RPD). While the primary coping is permanently cemented to the support, the secondary telescopic crown is attached to the prosthesis. Copings protect the support from decay and thermal irritation and provide the retention and stabilization of the secondary crown. The conical arrangement of the contact walls creates a compressive stress between the surfaces, the foundation of which rests on the compression effect. This tension should be strong enough to hold the RPD in place. The retention and stability of the telescopic attachments are related to their number, the conical angles of the walls and their distribution in the dental arch.

The taper angle is usually 6 degrees [4]. Increasing the taper of the copings decreases the retention, and the reduction of the taper increases the retention by increasing the friction force between the primary and secondary crowns [3]. The taper angle should be determined according to the patient, when the support length is insufficient, a taper angle of 2-5 degrees should be used. When the primary and secondary crowns come into contact, the splinting effect occurs in the restoration. Telescope retained prostheses (TRP) are a safe and effective type of prosthesis in carefully selected cases [10].

Susanne Merk et al. made secondary crowns from 3 different types of PEEK material (pre fabric block, pressed pellet, pressed granule) on $0,1,2$ degree zirconium primary crowns and tested retention. They found that the retention strength was greater in the secondary crowns group produced from industrial pellets pressed on a $0^{0}$ angle primary [44]. Veronika Stock et al. found that the retention of crowns (Co-Cr, $\mathrm{ZrO} 2$, galvanic) primary peek crowns of three different materials on the primary crown of $0,1,2$ degrees was sufficient regardless of the type of material and the taper angle. This is inconsistent with previous studies indicating that the taper angle affects conservatism [45]. Franz Sebastian Schwindling et al. made zirconia secondary crowns on 1 and 2 degree zirconia primary crowns and tested the retention force. They stated that CAD / CAM telescopic zirconia crowns provide sufficient retention when made with correct milling and care [46]. Stefan Baser et al. compared the intraoral and extraoral retention of telescopic crowns and found a correlation in the retention force plot.

The average taper angle is 6 degrees. Retention decreases as tapering decreases in copings. The angulation below this degree increases the friction force of the retainer crown. If the contact interfaces between the crowns are limited by the abutment interfaces. Angles between $2-5$ degrees are sufficient to provide adequate retention. For each patient, the wall angulations of the primary crown for the elements of the removable prosthesis are pre-evaluated and adjusted. Stabilisation and retention of telescopic attachments, number of telescopic crowns, taper angles and distributions in restorations involving full arch are related. The wall taper angulation is usually 6 degrees. Increasing the coping taper angle causes a decrease in the retention between the elements of the telescopic system. Recent in vitro studies in the lateral show that a retention force of 3-7 $\mathrm{N}$ 'per attachment is acceptable [1].

TRP can be preferred in tooth-tissue supported and only tooth supported cases. TRP can be compared functionally with fixed prostheses, the principles of fixed prostheses are taken into account in the distribution of supports. In this respect, TRP should be preferred in the presence of evenly distributed teeth on both sides of the arch. One of the important advantages of TRPs is that corrections can be made. In cases where the prognosis of the remaining teeth is in doubt. Splinting with a fixed prosthesis may be problematic. The telescopic prosthesis is a better alternative for this type of patient, because in the event of a local tooth loss, the telescopic prosthesis can be repaired without the need to renew the entire superstructure. The patient can remove TRP by separating the primary and secondary crowns. There is plenty of evidence that telescopic crowns improve oral hygiene and periodontal health. Gingival areas and prosthesis can be easily cleaned after the prosthesis is removed from the mouth. The splinting effect of TRP's support teeth also increases periodontal health $[3,10]$.

Telescopic prostheses are an alternative treatment approach in well-selected cases with the advantage of splinting and retention similar to fixed prostheses and ease of repair. Telescopic prostheses transmit occlusal forces along the long axis of the tooth. The telescopic prosthesis can be removed by the patient and can be cleaned easily, braces are advantageous in terms of periodontal health and oral hygiene. In addition to technical and clinical principles, planning and indication should also be done carefully. Five to ten years long term Many studies indicate that TRPs show less loss compared hooked RDP and precision attachment prostheses [47].

Owal et al. found that $25 \%$ of the RPD performed was removed in the 1st year due to insufficient retention and stability of the prosthesis [48]. During the first tooth extraction or tooth loss period, the alveolar bone is damaged rapidly in all dimensions, retention and stability loss occur [48,49]. Preserving the bone level or size around teeth, tooth roots and implants is extremely effective [50]. Overinvolved tooth roots serve to preserve the alveolar crest [51]. It not only serves to preserve the bone but also the retention and the stability of the prosthesis [52]. The telescopic prosthesis is mainly used to transmit occlusal forces from the artificial teeth to the abutment without exerting damaging forces on the alveolar protrusion. Moreover, the added quality with the telescopic RPD is proprioception because, as far as possible, the periodontal fibers of the retained teeth in the mouth give patients a sense of discrimination of various pressure-like sensations [28,53]. Successful use of the telescopic prosthesis depends on the effective completion and maintenance of the endodontic treatment of the abutment teeth. If the abutment is tooth, correct diagnosis is very important, current periodontal status, radiographic examination, level of the remaining bone, evaluation of any systemic disease of the patient, oral hygiene status and prognosis, all of these should be taken into consideration. Accurate diagnosis and skilled endodontic treatment are highly successful [54,55].

According to a retrospective study performed on 72 patients with 75 tooth-supported telescopic prostheses, $7 \%$ loss was observed in supporting teeth after 3.8 years of observation [21]. In another study where 554 TRPs were examined for 5 years, it was determined that $4.7 \%$ of the prostheses lost their function and $3.8 \%$ of the supporting teeth were lost. The survival rate after 5 years is $95 \%$ in prosthesis and support teeth. At least 4 supporting teeth are recommended in TRPs. Facial repair was required in $26.9 \%$ of the prostheses, lining in $34.8 \%$, and desimentation in the primary crown in $20.6 \%$ [17]. According to the results of another study comparing RDPs with rigid precision attachment, telescopic crown attachment and hook attachment in terms of the stresses on the support tooth and prosthesis base and the movements of the denture base, rigid precision attachment and telescopic attachment prostheses create more stress on the final support tooth compared to the hook attachment prosthesis. . The rigid precision attachment prosthesis, on the other hand, creates more stress on the final support tooth than the telescope retained prosthesis. The stress on the prosthesis base is less in the rigid precision attachment and telescope attachment prosthesis. Rigid connection and cross-arc stabilization are recommended to reduce the movement of the prosthetic base [56]. 


\section{Conclusion}

The article describes of telescopic crown types and clinical factors, as well as design and indication principles, were discussed. Stabilization of risky teeth with fixed splinted restorations is not generally recommended due to associated risk factors such as eventual localized abutment failure. Removable telescopic prostheses can be preferred because they can be removed and repaired without reconstructing the entire restoration. The retention and splinting properties of detachable telescopic restorations can be as effective as FPDs. Internal telescopic copings can be cemented as separate crowns to facilitate the procedure. Telescopic restorations can be taken by the patient for cleaning and easy access to the entire marginal periodontal area of the abutments. This promotes effective home care and oral hygiene.

\section{Acknowledgement}

The author has no conflicts of interest to disclose.

\section{References}

[1] Bayer S, Stark H, Gölz L, et al. Telescopic crowns: Extra-oral and intra-oral retention force measurement -in vitro/in vivo correlation. Gerodontology. 2012;29.

[2] Hakkoum MA, Wazir G. Telescopic Denture. Open Dent J. 2018;12.

[3] Langer A. Telescope retainers and their clinical application. J Prosthet Dent. 1980;44.

[4] Langer A. Telescope retainers for removable partial dentures. J Prosthet Dent. 1981;45.

[5] Langer A. Tooth-supported telescope restorations. J Prosthet Dent. 1981;45.

[6] Behr M, Hofmann E, Rosentritt M, et al. Technical failure rates of double crown-retained removable partial dentures. Clin Oral Investig. 2000;4.

[7] Reitz P V., Weiner MG, Levin B. An overdenture survey: Preliminary report. J Prosthet Dent. 1977;37.

[8] Schweitzer JM, Schweitzer RD, Schweitzer J. The telescoped complete denture: A research report at the clinical level. J Prosthet Dent. 1971;26.

[9] Dhir RC. Clinical assessment of the overdenture therapy. J Indian Prosthodont Soc. 2005;5.

[10] Langer Y, Langer A. Tooth-supported telescopic prostheses in compromised dentitions: A clinical report. J Prosthet Dent [Internet]. 2000;84:129-132. Available from: https://linkinghub.elsevier.com/retrieve/pii/S0022391300130501.

[11] Stančić I, Jelenković A. Retention of telescopic denture in elderly patients with maximum partially edentulous arch. Gerodontology. 2008;25.

[12] Besimo C, Graber G. A new concept of overdentures with telescope crowns on osseointegrated implants. Int J Periodontics Restorative Dent $1994 ; 14$.

[13] Besimo C, Graber G, Schaffner T. Hybrid prosthetic implant supported suprastructures in edentulous mandible. Conus crowns and shell-pinsystems on HA-Ti-implants. 2. Prosthetic construction principles. ZWR. 1991.

[14] Panek H. Z_Year Clinical Observation of Telescopic Anchors Applied in Removable Dentures - Case Report Z_letnie obserwacje kliniczne zaczepów teleskopowych zastosowanych w protezach ruchomych - opis przypadku. 2007.

[15] Kiyama M, Shiba A. Studies on Retentive Force of Conical Telescopic Double-Crown. Part 1. Retentive Force of Conical Telescopic DoubleCrown Related to Materials and Taper Angle and Height of Cone and Load. Nihon Hotetsu Shika Gakkai Zasshi. 1994;38.

[16] Güngör MA, Artunç C, Sonugelen M, et al. The evaluation of the removal forces on the conus crowned telescopic prostheses with the finite element analysis (FEA). J Oral Rehabil. 2002;29.

[17] Wöstmann B, Balkenhol M, Weber A, et al. Long-term analysis of telescopic crown retained removable partial dentures: Survival and need for maintenance. J Dent. 2007;35

[18] Breeding LC, Dixon DL, Sadler JP, et al. Mechanical considerations for the implant tooth-supported fixed partial denture. J Prosthet Dent. 1995;74:487-492.

[19] Ohkawa S, Okane H, Nagasawa T, et al. Changes in retention of various telescope crown assemblies over long-term use. J Prosthet Dent. 1990;64.

[20] Hultén J, Tillström B, Nilner K. Long term clinical evaluation of conical crown retained dentures. Swed Dent J. 1993;17.

[21] Widbom T, Löfquist L, Widbom C, et al. Tooth-Supported Telescopic Crown-Retained Dentures: An up to 9-Year Retrospective Clinical Follow-up Study. Int J Prosthodont. 2004;17.

[22] Shiba A, Suzuki K, Hoshino M, et al. Telescopic denture and clasp for the partially edentulous jaw. Hotetsu Rinsho. 1983;Spec No.

[23] Güngör MA, Artunç C, Sonugelen M. Parameters affecting retentive force of conus crowns. J Oral Rehabil. 2004;31.

[24] Minagi S, Natsuaki N, Nishigawa G, et al. New telescopic crown design for removable partial dentures. J Prosthet Dent. $1999 ; 81$.

[25] Wenz HJ, Lehmann KM. A telescopic crown concept for the restoration of the partially edentulous arch: the Marburg double crown system. Int J Prosthodont. 1998;11:541-550.

[26] Kazokollu FŞ, Akaltan F. Strain characteristics of Marburg double crown-retained implant overdentures compared with bar and ball-retained implant overdentures, with and without a rigid major connector. J Prosthet Dent. 2014;112.

[27] Prakash V, Parkash H, Gupta R. Fixed removable prosthesis employing Marburg double crown system. J Indian Prosthodont Soc. 2008;8.

[28] Wenz HJ, Hertrampf K, Lehmann KM. Clinical Longevity of Removable Partial Dentures Retained by Telescopic Crowns: Outcome of the Double Crown with Clearance Fit. Int J Prosthodont. 2001;14.

[29] Krennmair G, Weinländer M, Krainhöfner M, et al. Implant-supported mandibular overdentures retained with ball or telescopic crown attachments: A 3-year prospective study [Implantatgetragene Deckprothesen im Unterkiefer auf Kugelgeschieben oder Teleskopkronen - Eine prospektive Dreijahresstudie]. Implantologie. 2006;14

[30] Hegazy S, Emera R. Assisted Complete Mandibular Overdentures : Invitro Stress. 2014;

[31] Langer A. Combinations of diverse retainers in removable partial dentures. J Prosthet Dent. 1978;40.

[32] Isaacson GO. Telescope crown retainers for removable partial dentures. J Prosthet Dent. 1969;22.

[33] Weaver JD. Telescopic copings in restorative dentistry. J Prosthet Dent. 1989;61.

[34] Stern N, Revah A. Repairing a crown and sleeve-coping prosthesis. J Prosthet Dent. 1980;43.

[35] Bayer S, Al-Mansour R, Grüner M, et al. In-vitro-Verschleiß von Aktivierungselementen für Teleskopkronen. ZWR - Das Dtsch Zahnärzteblatt. 2008;117.

[36] Akagawa Y, Seo T, Ohkawa S, et al. A new telescopic crown system using a soldered horizontal pin for removable partial dentures. J Prosthet Dent. 1993;69.

[37] Zierden K, Kurzrock L, Wöstmann B, et al. Nonprecious Alloy vs Precious Alloy Telescopic Crown-Retained Removable Partial Dentures: Survival and Maintenance Needs. Int J Prosthodont. 2018;31.

[38] Chee W, Jivraj S. Connecting implants to teeth. Br Dent J. 2006;201.

[39] Ericson A, Nilsson B, Bergman B. Clinical results in patients provided with conical crown retained dentures. Int J Prosthodont. 1990;3.

[40] Tang ZY, Jiang Y De. Study of telescopic denture in patients with defect of dentition. J Dalian Med Univ. $2008 ; 30$. 
[41] GL H. Clinical Application of CSC Telescopic Denture with Magnetic Attachment in Treating Class III Molar Furcation Involvement-A Case Report. Int J Dent Oral Heal. 2020;6.

[42] Weischer T, Mohr C. Implant-Supported Mandibular Telescopic Prostheses in Oral Cancer Patients: An up to 9-Year Retrospective Study. Int J Prosthodont. 2001;14.

[43] Rinke S, Ziebolz D, Ratka-Krüger P, et al. Clinical Outcome of Double Crown-Retained Mandibular Removable Dentures Supported by a Combination of Residual Teeth and Strategic Implants. J Prosthodont. 2015;24.

[44] Merk S, Wagner C, Stock V, et al. Suitability of secondary PEEK telescopic crowns on zirconia primary crowns: The influence of fabrication method and taper. Materials (Basel). 2016;9.

[45] Stock V, Schmidlin P, Merk S, et al. PEEK Primary Crowns with Cobalt-Chromium, Zirconia and Galvanic Secondary Crowns with Different Tapers-A Comparison of Retention Forces. Materials (Basel). 2016;9.

[46] Schwindling FS, Dittmann B, Rammelsberg P. Double-crown-retained removable dental prostheses: A retrospective study of survival and complications. J Prosthet Dent. 2014;112.

[47] Beschnidt SM, Chitmongkolsuk S, Prull R. Telescopic crown-retained removable partial dentures: review and case report. Compend. Contin. Educ. Dent. 2001

[48] Öwall B, Bieniek KW, Spiekermann H. Removable partial denture production in western Germany. Quintessence Int (Berl). 1995;26.

[49] McGarry TJ, Nimmo A, Skiba JF, et al. Classification system for complete edentulism. J Prosthodont. $1999 ; 8$.

[50] Atieh MA, Ibrahim HM, Atieh AH. Platform Switching for Marginal Bone Preservation Around Dental Implants: A Systematic Review and Meta-Analysis. J Periodontol. 2010;81.

[51] Bhagat TV, Walke AN. Telescopic Partial Dentures-Concealed Technology. J Int oral Heal JIOH. $2015 ; 7$.

[52] Wöstmann B, Balkenhol M, Kothe A, et al. Dental impact on daily living of telescopic crown-retained partial dentures. Int J Prosthodont [Internet]. 2008;21:419-421. Available from: http://www.ncbi.nlm.nih.gov/pubmed/18950064.

[53] Dittmann B, Rammelsberg P. Survival of abutment teeth used for telescopic abutment retainers in removable partial dentures. Int J Prosthodont [Internet]. 2008;21:319-321. Available from: http://www.ncbi.nlm.nih.gov/pubmed/18717090.

[54] Stanley HR. Management of the aging patient and the aging pulp. J Calif Dent Assoc. 1977;5:62-64.

[55] Goodis HE, Curtis D. Endodontic Considerations When Fabricating Overdentures. Gerodontology. 1990;9:25-28.

[56] Saito M, Miura Y, Notani K, et al. Stress distribution of abutments and base displacement with precision attachment- and telescopic crownretained removable partial dentures. J Oral Rehabil. 2003;30. 